\title{
What Does a Positive Test Result Really Mean?
}

\author{
Jie W. Weiss \\ California State University, Fullerton
}

One of our most important tasks is communicating health risks to the public. We do a poor job of that, primarily because the metric we use, probability, is not well understood. The misunderstanding does not arise merely because ordinary folks are poorly educated in this regard. Indeed, as Gird Gigerenzer (2002) of the Max Planck Institute in Berlin has repeatedly demonstrated, health professionals are no less confused by probabilities than laypersons.

Try to answer this question, which has befuddled many experienced physicians. Suppose a patient tests positive on the Haemoccult test for colorectal cancer. This is pretty alarming news, isn't it? The test is used as a preliminary screen. It has a sensitivity rate of $50 \%$, which means that if a person has the disease, the test result will be positive half the time. The test has a false positive rate of only $3 \%$ (which is a specificity rate of $97 \%$ ). The prevalence rate of the cancer within the population to which this person belongs is $0.3 \%$. What is your estimate of the probability that the patient who just tested positive actually has colorectal cancer? About half the doctors said it was close to $50 \%$. Does that seem plausible to you? Well, it's about ten times the actual probability, which is around $5 \%$.

To understand that answer, approach the problem not with confusing probabilities, but with what Gigerenzer calls "natural frequencies". Consider a population of 10,000 people like the patient and imagine they all are tested. Of those, $30(=.3 \%)$ have the cancer. The sensitivity of the test $(50 \%)$ tells us it will yield accurate positive results for 15 of them. The false positive rate (3\%) tells us the test will yield inaccurate positive results for 299 of the 9970 people who do not have the cancer. So of the 314 people who test positive, 15 (4.7\%) actually have the cancer. If the conversation focuses on the whole numbers and foregoes probabilities, this arithmetic is not too complex for most people to grasp.

The critical element in the analysis, the one human intuition often ignores, is the prevalence rate for the disease. Not coincidentally, the diseases for which screening tests are recommended for asymptomatic clients generally have low prevalence rates. Therefore, if the disease is infrequent, even a slightly imperfect test (and all tests have non-zero rates for false positives and false negatives) is likely to lead to excessive worry. Some of the consequences are medical - invasive follow-ups, stress-induced conditions- and others are psychological. Receiving bad news about a dreaded disease can inspire depression and drastic life changes.

Even for an extremely accurate multiple-test battery such as that used for AIDS testing - both error rates are on the order of $.1 \%$. Therefore positive results for a member of a low-risk group imply only about a 1 in 2 chance that the person has the disease. Gigerenzer recounts tragic tales of suicides following the delivery of positive AIDS test results. He argues that presenting test results in a way that excessively frightens patients is more than poor communication; it is a serious ethics violation. That is why professionals always need to consider the negative consequences of delivering the news of a positive test result, and to remind the patient of the probability that the test result was a false positive.

(C) 2011 Californian Journal of Health Promotion. All rights reserved

References

Gigerenzer, G. (2002). Calculated risks: how to know when numbers decide you. New York: Simon \& Schuster. 